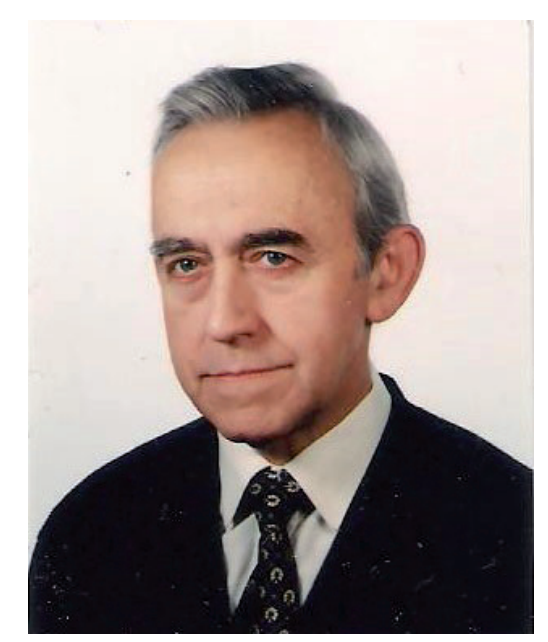

\title{
RECOLLECTIONS OF WORK AT THE FLIGHT HYGIENE AND SAFETY DEPARTMENT AT MIAM IN THE YEARS
} 1972-1998

Zbigniew KOTER

Source of support: Own sources

Author's address: Z. Koter, Warsaw, Poland, e-mail: zbigniew-koter@wp.pl

Abstract: The author, as a pharmacist, presents research and organizational problems of a scientific institute dealing with scientific research and application tests in the field of flight hygiene and safety in the years 1973-1998. He also describes expert activities and procedures for investigating air accidents from this perspective.

Keywords: Pharmacology, occupational health, flight safety, plane crashes from a pharmacologist's perspective 


\section{INTRODUCTION}

My first contact with the Military Institute of Aviation Medicine (MIAM) dates back to 1973, when my superior, head of the Department of Toxicology at the Medical Academy in Warsaw, Prof. Władysław Rusiecki commissioned me to perform tests to determine whether the level of carbon monoxide in the pilot's organism can be determined in the section material collected several dozen hours after a crash. The research carried out with the technical assistance of the MIAM Flight Hygiene and Safety Department resulted in a joint publication in the magazine "Medycyna Lotnicza" ("Aviation Medicine"). My participation in these studies was positively evaluated, as a result of which I was offered the position of an Assistant Professor at the Flight Hygiene and Safety Department. In 1974, I started working at the toxicology laboratory of the Flight Hygiene and Safety Department.

\section{THE FLIGHT HYGIENE AND SAFETY DEPARTMENT}

The Flight Hygiene and Safety Department (FHSD) had four laboratories dealing with pathomorphology, toxicology, hygiene of flight and technical personnel clothing as well as food hygiene of the above mentioned personnel. The first basic activity of the Department was the research conducted for the Military Committee for Investigation of Aviation Accidents. Pathologists from the Department were directed to the place of the plane crash each time in order to conduct an external and internal medical examination in order to exclude or determine the causes of the crash taking into account the disease changes in the pilot's body and to collect biological material for further examination at the Department. The collected material was delivered to the toxicology laboratory for biochemical and toxicological tests, where I performed tests for presence of alcohol in blood, carbon monoxide in the pilot's organism and carbohydrate profile in selected organs. Protocols from these examinations were sent to the accident investigation committee. The second part of the material was transferred to the pathomorphology laboratory for further histological examination.

\section{RESEARCHES FOR FLIGHT HYGIENE AND SAFETY}

Apart from the aforementioned research resulting from aviation emergency situations, the Department conducted research within the framework of the scientific plan of the Military Institute of Aviation Medicine. The research was completed in 1977 with a publication entitled: "Changes in the activity and morphological picture of the endocrine system under conditions of altitude hypoxia, high and low temperatures and combined impact of these factors". They were honored with the first place in the MIAM Commander's contest for scientific papers.

In the following years, research on the influence of hypoxia on the body was continued at the Department. It was appreciated by the Minister of National Defence, who in 1979 awarded the team, which I was a part of, with a second-degree team award in military medicine for experimental and clinical elaboration of effects of hypoxia on the human body.

In 1980, after presentation of the doctoral dissertation entitled "The influence of ethanol and caffeine on tolerance of acute altitude hypoxia" to the Scientific Council of the Pharmaceutical Department of the Medical Academy I obtained a PhD degree in pharmaceutical sciences.

In the years 1978-1990, the Flight Hygiene and Safety Department actively participated in interdisciplinary research conducted by selected research institutes of the Jagiellonian University, the University of Warsaw, the Nicolaus Copernicus University in Toruń, the Military Medical Academy and the University of Physical Education in Gorzów Wielkopolski, within the framework of the governmental programme R III-14 on the influence of biological rhythms on human and animal organisms. The FHSD conducted research on the influence of biological rhythms on the human body in the aspect of work in the air. I have also actively participated in these studies. As a result of this research, in 1985 the Minister of National Defence awarded the following team: prof. Krzysztof Kwarecki, doc. Władysław Święcicki, dr Zbigniew Koter and mgr Krystyna Zużewicz, the first-degree award in military medicine for "A series of studies concerning disturbances of biological rhythms in the aspect of flight safety".

In the years 1979-1980, the toxicology laboratory conducted hygienic and toxicological tests in the cabin of the TS-11 "Iskra" training aircraft and on behalf of the Communication Equipment Factory in Świdnik - hygienic and toxicological tests in the cabins of the "Kania" and "Sokół" helicopters. These tests were necessary to present both helicopters manufactured in Świdnik for approval. For the research entitled "Experimental works concerning new aircraft manufactured in Poland", in 1980 the FHSD received the first prize by the Commander of MIAM in a scientific works contest. 


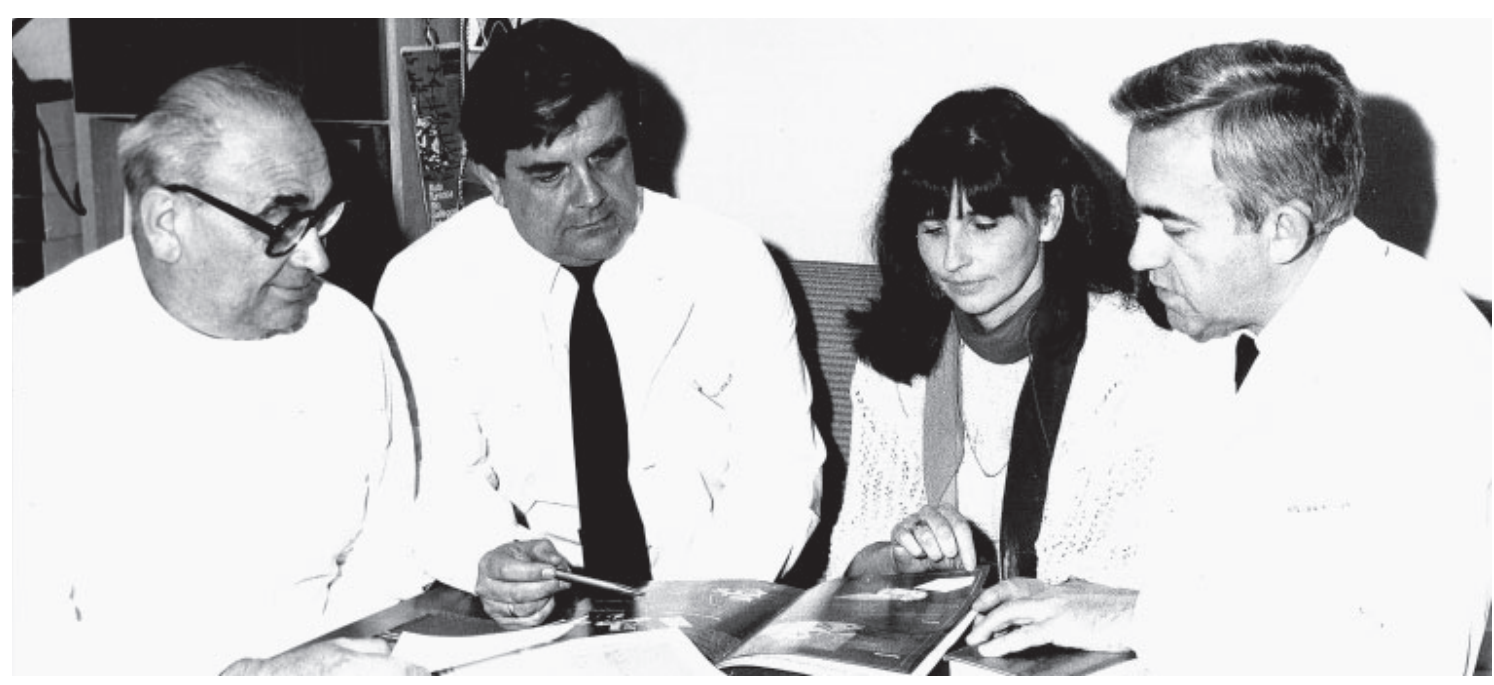

Fig. 1. Team of the Flight Hygiene and Safety Department (FHSD) (from left to right: prof. Władysław Święcicki, prof. Krzysztof Kwarecki, mgr Krystyna Zużewicz i dr Zbigniew Koter).

\section{INTERNATIONAL RESERCHES FOR INTERKOSMOS}

In 1978 a cooperation with scientists was established with the Institute of Medical and Biological Issues in Moscow, which resulted in sending to Moscow a team of 3 people led by me, in order to undertake research on animals present in space under conditions of changed gravity on Sputnik 1138. A further part of the tests was carried out at the FHSD. I have presented the results of these studies in 1980 at the International Scientific Congress INTERKOSMOS in Dresden in the form of two scientific reports. A report on the completion of this work, entitled "The cycle of research on the influence of variable gravitational conditions on selected biochemical and physiological reactions" was awarded in 1981 the first prize by the Commander of MIAM in a scientific works contest.

In 1982, the FHSD and the Department of Pathophysiology organized two camps for pilots with high BMI in the "Gronik" and "Mrągowo" fitness centers. The aim was to improve the condition of flying personnel, especially overweight pilots, through appropriate diet profiling and fitness training in order to reduce the body weight. At that time I conducted biochemical research in the name of FHSD, determining lipid profile, insulin level and carbohydrate profile in pilots before and after the camps, during which a properly profiled diet combined with increased physical effort was applied during training in the hall and during training in the mountains. A scientific paper summarizing the above mentioned research entitled "The cycle of research on the state of health of flying and technical personnel" was awarded with a diploma for taking the first place in the competition of scientific works of the Military Institute of Aviation Medicine.

At the beginning of the 1990s, after the personnel changes at the Department and the expiry of the governmental program R III-14 on the influence of biological rhythms on the human body in the aspect of work in the air, the research focused on the influence of hypoxia on the disturbances in the rhythm of daily melatonin secretion in the pineal gland. Some of the research was conducted in cooperation with the Faculty of Biology at the University of Warsaw. This research resulted in several scientific publications in Polish and foreign journals.

In 1998 I left my job at Flight Hygiene and Safety Department at MIAM and started teaching at the Department of Toxicology at the Medical Academy in Warsaw.

\section{AUTHORS' DECLARATION:}

Study Design: Zbigniew Koter; Data Collection: Zbigniew Koter; Manuscript Preparation: Zbigniew Koter. The Author declares that there is no conflict of interest.

Cite this article as: Koter Z. Recollections of Work at The Flight Hygiene and Safety Department at Miam in The Years 19721998. Pol J Aviat Med Bioeng Psychol 2017; 23(3-4): 50-52. DOI: 10.13174/pjambp.20.12.2018.08 